\title{
Passive tactile feedback facilitates mental rotation of handheld objects
}

\author{
Maryjane Wraga, Monique Swaby, and Catherine M. Flynn \\ Smith College, Northampton, Massachusetts
}

\begin{abstract}
Mental rotation of objects improves when passive tactile information for the rotating object accompanies the imagined rotation (Wraga, Creem, \& Proffitt, 2000). We examined this phenomenon further using a withinsubjects paradigm involving handheld objects. In Experiment 1, participants imagined rotating an unseen object placed on their upturned palms. The participants were faster at mental rotation when the object was rotated on their palm than when the object remained stationary. Experiment 2 tested whether the performance advantage would endure when the participants received tactile information for only the start- and endpoints of the rotation event. This manipulation did not improve performance, relative to a stationary control. Experiment 3 revealed that ambiguous tactile information, continuous with the rotation event but independent of object shape, actually degraded performance, relative to a stationary control. In Experiment 4, we found that continuous tactile rotation discrepant from imagined object movement also hindered performance, as compared with continuous tactile information aligned with imagined object movement. The findings suggest a tight coupling between tactile information specifying continuous object rotation and the corresponding internal representation of the rotating object.
\end{abstract}

The ability to mentally rotate objects is essential to a variety of human spatial reasoning tasks, from planning how to pack the trunk of a car to solving geometry problems. The classic self-congruence paradigm of Shepard and Metzler (1971), in which participants viewed a misaligned pair of objects and decided whether they were identical, established that people could perform mental transformations of objects. Shepard and Metzler's study also revealed an important parallel between imagined and real-world transformations of objects: The amount of time it took participants to perform the self-congruence task increased monotonically with the angular disparity between objects. This finding suggests that individuals mentally rotate objects in the same manner in which they physically rotate objects, despite the fact that mental space need not adhere to the laws of physics. Subsequent studies have replicated the monotonic response time (RT) function with a variety of stimuli, including alphanumeric characters (e.g., Corballis \& McMaster, 1996; Jolicœur \& Cavanagh, 1992) and depictions of body parts, such as the hands and feet (e.g., Kosslyn, DiGirolamo, Thompson, \& Alpert, 1998; Parsons, 1987).

One interesting subclass of the mental rotation paradigm involves tactile discriminations, in which participants update the orientation of an unseen object actively felt by the hand. Many of these tasks have produced monotonic RT functions similar to those produced during mental rotation of visual stimuli (e.g., Carpenter \& Eisenberg, 1978; Dellantonio \& Spagnolo, 1990; Marmor \& Zaback, 1976; Robert \& Chevrier, 2003); they com- monly are referred to as tactile mental rotation tasks (e.g., Prather \& Sathian, 2002). For example, Carpenter and Eisenberg tested both sighted and blind participants on a tactile mental rotation task in which they had to decide whether letters explored actively with the hand were normal or mirror reversed. The time it took the participants to make the normal/mirror-reversed discrimination was monotonically related to the initial orientation of the letter with respect to its canonical upright. Dellantonio and Spagnolo obtained similar results in sighted individuals who made normal/mirror-reversed discriminations while actively exploring abstract pin configurations with their hands. A more recent study by Robert and Chevrier demonstrated that participants who actively explored more complex, 3-D objects such as those originally created by Shepard and Metzler (1971) also could perform tactile mental rotation, albeit at an overall cost to RTs larger than that for simpler shapes.

Tactile mental rotation tasks also can be performed when unseen objects are felt through passive touch, in which the tactile stimulation stems from an external source, rather than from one's hand (Prather \& Sathian, 2002; Prather, Votaw, \& Sathian, 2004). Prather and Sathian used a paradigm in which a raised "J" stimulus was pressed onto the tip of a participant's upturned index finger, at different orientations. The researchers found that time to identify the letter increased monotonically with the angle of disparity between the canonical upright of the letter and the long axis of the finger when they coincided with each other. Interestingly, when the hand was rotated $90^{\circ}$ counter- 
clockwise from that position, the participants produced the shortest RTs when the stimulus was misaligned $90^{\circ}$ clockwise from the long axis of the finger. This finding suggests that mental rotation of tactile stimuli occurs independently of the reference frame of the hand and, thus, may be based on general, rather than modality-specific, processes.

Given the similarities between tactile mental rotation performance and visual mental rotation performance, an important question is whether they share common processing mechanisms. One account involves visual imagery (Prather et al., 2004). Visual imagery is thought to play a major role in visual mental rotation (e.g., Shepard \& Cooper, 1982; cf. Pylyshyn, 1973). Recent support for this claim has come from functional neuroimaging studies, which have shown activation in visual-processing regions during visual mental rotation tasks (e.g., Cohen et al., 1996; Kosslyn et al., 1998; Wraga, Shephard, Church, Inati, \& Kosslyn, 2005). Sathian and colleagues have hypothesized that the tactile feedback participants receive during active and passive haptic mental rotation tasks may be translated into visual format for mental rotation (Prather \& Sathian, 2002; Prather et al., 2004; Sathian, 2005). In the present study, we explored this issue using a behavioral paradigm. Specifically, we focused on the degree to which passive tactile information from a physical object may influence mental rotation of previously seen objects.

Passive tactile feedback engages cutaneous receptors in the human hand, which specify information about the geometric properties of an object, such as its size, shape, and the spatial relations of its parts (Lederman \& Klatzky, 1997; Loomis \& Lederman, 1986). Passive tactile feedback is thought to play a major role in the creation of static (i.e., nonmoving) internal representations of object shape (Voisin, Benoit, \& Chapman, 2002; Voisin, Lamarre, \& Chapman, 2002). For example, Voisin, Lamarre, and Chapman systematically manipulated the presence and absence of proprioceptive and tactile feedback on participants' ability to discriminate size differences of as little as $1^{\circ}$ between 2-D Plexiglas angle pairs. Performance was optimal when the participants utilized both proprioceptive and tactile information through active exploration of the angles. However, when tactile information was blocked, via local anesthesia to the participant's index finger, the decrement in performance was equivalent to that created when proprioceptive information was absent through passive touch exploration of the angles. The researchers concluded that both sources of information combine in an integrative fashion to inform spatial discrimination. Interestingly, the majority of participants in the passive condition reported using the tactile feedback to generate internal visual representations of the angles.

The influence of tactile information on dynamic (i.e., moving) transformations of an object's internal representation is less clear. One promising finding in the literature is that mental rotation of previously seen objects improves when passive tactile feedback for the rotating object accompanies the imagined rotation (Wraga, Creem, \& Proffitt, 2000). In a preliminary study, Wraga et al. (2000) found that participants who received passive feedback of a rotating object on their upturned palm while imagining the objects rotating were significantly faster at updating the object's configuration than were participants who imagined rotating an object that remained stationary on their palm. Wraga et al. (2000) hypothesized that the tactile information received during passive rotation improved the internal consistency of the visual representation of the rotating object by providing online location of the rotating object with respect to the hand during the entire rotation event. However, at least two alternative hypotheses exist. One is that the start- and endpoints of the rotating object, and not the continuous transformation thereof, facilitated updating performance. The other is that the tactile information present during the rotating object event facilitated performance in its own right, independently of any influence on the internal representation of the object.

The present study was designed to explore these issues further with a mental rotation task involving handheld objects. Experiment 1 established a within-subjects paradigm for testing effects of passive tactile information on mental rotation performance. Experiment 2 demonstrated that discrete tactile information specifying the start- and endpoints of the rotating object was not sufficient for improving mental rotation performance. Experiment 3 demonstrated that continuous tactile information independent of the rotating object's shape actually hindered mental rotation performance. In Experiment 4, continuous tactile feedback matching the object's shape but discrepant from the object's prescribed rotation hindered mental rotation performance, as compared with continuous tactile feedback in sync with the prescribed rotation.

\section{GENERAL METHOD}

This section will describe aspects of the experimental method common to the four experiments. Details specific to the individual experiments will be included within their corresponding sections.

\section{Materials}

The objects used were two $8 \times 8 \mathrm{~cm}$ wooden puzzle pieces, one depicting a cat, the other a dog (see Figure 1). Each object had a 2-cm diameter, 2.8-cm length wooden knob affixed to its center. We recorded RTs using a Timex chronographic stopwatch.

\section{Procedure}

Each participant performed in two conditions, each with a different object. The participant stood with her nondominant hand extended in front of her, palm facing up, elbow touching the side of her body. The experimenter placed one object in the participant's hand and pointed out the location of the four components of the object (e.g., "head," "feet," "tail," and "chest") with respect to "top," "bottom," "left," and "right" locations. The participant was given as much time as necessary to memorize the locations of the components with eyes open. She then was tested on the locations of the four components with eyes closed. Criteria for learning were achieved if the participant correctly identified each component within $1 \mathrm{sec}$. The experimenter next explained the task. For the control condition, the object remained stationary on the participant's palm for the duration of the trials. The participant was instructed to imagine rotating the object flat on her palm in a clockwise direction and then had to decide which component of the object appeared in a prescribed location. Before testing began, the participant was blindfolded. Each trial consisted of a ro- 


\section{A}

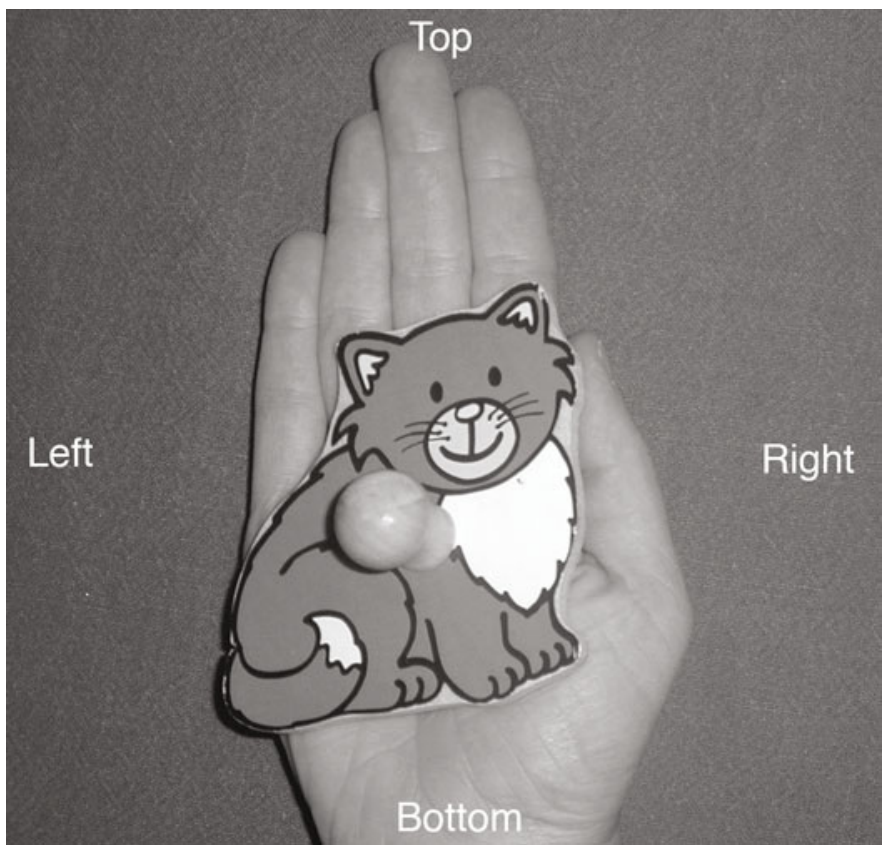

B

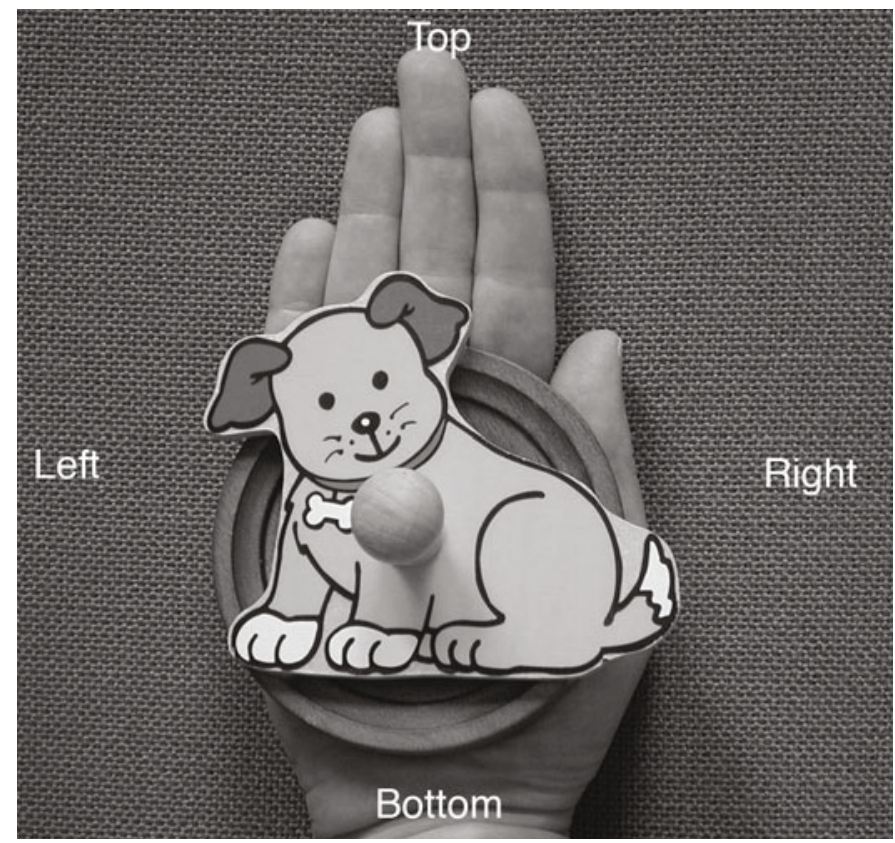

Figure 1. (A) One of the stimuli used in Experiments 1, 2, and 4, positioned on a person's hand. The four locations given indicate positions with respect to the hand. For this particular configuration, the participant would memorize that the cat's "head" faces the top; its "chest" faces the right; its "feet" face the bottom; and its "tail" faces the left. (B) The other stimulus used, positioned on a person's hand and mounted in the object-on-disk arrangement in Experiment 3.

tation magnitude $\left(0^{\circ}, 90^{\circ}, 180^{\circ}\right.$, or $\left.270^{\circ}\right)$, followed by a location on the hand-for example, " $90^{\circ}$, what is on the left?" The participant responded by naming a component of the object. She was instructed to respond as quickly and accurately as possible. The experimenter recorded RTs and responses. RT was measured from the end of the experimenter's question to the onset of the participant's response. At the completion of the first set of trials, the experimenter removed the object from the participant's palm and placed the other object onto the palm. The procedure for the next condition then commenced (see the individual experiments for details of other conditions). 


\section{Design}

The order of the two objects was counterbalanced across tasks. Because the objects contained similar parts, we used two different orientations with respect to the participant's palm, to eliminate reliance on memory strategies across conditions. The objects appeared either at a $0^{\circ}$ orientation, in which the "head" of the object was aligned with the "top" position of the hand (see Figure 1), or at a $90^{\circ}$ orientation, in which the head of the object was rotated $90^{\circ}$ clockwise and aligned with the "right" position of the hand. The orientation of the object was counterbalanced across task. ${ }^{1}$ Order of task was counterbalanced across participants. The four locations on the hand (e.g., top, bottom, left, and right) were matched with each of the rotation magnitudes $\left(0^{\circ}, 90^{\circ}, 180^{\circ}\right.$, and $\left.270^{\circ}\right)$, for a total of 16 trials per task. The trials were presented randomly.

\section{Analyses}

We recorded RTs and calculated percentages of error for each condition in each experiment. ${ }^{2}$ Only RTs for correct trials were used in the analysis. RTs greater than 2.5 times the group mean for a particular condition were replaced by the condition group mean. This occurred for $<2 \%$ of the data. We performed a 2 (task order) $\times 2$ (task) $\times 4$ (rotation magnitude) mixed design ANOVA on the mean RT and error data, with task order as a between-subjects variable and task and rotation magnitude as within-subjects variables.

\section{EXPERIMENT 1}

The first experiment was a variation of Wraga et al.'s (2000) Experiment 6. They found that participants who received passive rotation of a rectangular block on their palm while imagining the object rotating were faster at updating subsequent components of the object, as compared with a control group who received no block-turning manipulation. In the present experiment, we applied the same manipulation using a within-subjects design to provide better control of between-group variability. The participants imagined rotating an object placed on the upturned palm of their dominant hand, while the object either remained stationary (control condition) or was rotated on their palm the prescribed rotation amount by the experimenter (tactile condition). On the basis of Wraga et al. (2000), we predicted that the participants' mental rotation performance would be significantly faster in the tactile condition than in the control condition.

\section{Method}

\section{Participants}

Twenty-three Smith College undergraduate females participated in the experiment as part of a research credit requirement. The data of 1 additional participant were excluded from the experiment for having more than a $50 \%$ error rate in at least one condition. All the participants were tested individually and were unaware of the hypothesis being tested.

\section{Materials}

See the General Method section.

\section{Procedure}

The procedure was identical to that described in the General Method section, with the following changes. The task described in the General Method section served as the control condition. The participants also performed in a tactile condition. For each trial, the experimenter rotated the object on the participant's palm while saying the required number of degrees of rotation. The experimenter rotated the object by turning its wooden handle the prescribed amount in a clockwise direction. Physical rotation of the object was timed so that its start- and endpoints coincided with the beginning and end of the announcement of rotation magnitude. After the participant's response and RT had been recorded, the experimenter removed the object from the participant's palm and rotated it in the air to its starting orientation before placing it back on the participant's palm. This measure ensured that the participant received tactile information for the rotating object only during the test trial itself. The next trial then commenced.

\section{Design}

See the General Method section.

\section{Results}

\section{Response Times}

Figure 2A shows mean RTs and standard errors for each condition as a function of rotation magnitude. The participants were significantly faster in the tactile condition $(M=1.74 \mathrm{sec})$ than in the control condition $(M=2.28 \mathrm{sec})$ $[F(1,21)=11.09, p<.003]$. The ANOVA also yielded a significant effect of rotation magnitude $[F(3,63)=22.02$, $p<.0001]$. Post hoc linear comparisons revealed an increase in RTs from $0^{\circ}$ to $90^{\circ}(p<.0001)$ and from $180^{\circ}$ to $270^{\circ}\left(p<.0001\right.$ ), but not between $90^{\circ}$ and $180^{\circ}$ ( $p=$ .905). We also found a significant condition $\times$ rotation magnitude interaction $[F(3,63)=3.36, p<.024]$. Contrast comparisons indicated that facilitated performance in the tactile condition occurred most significantly for $90^{\circ}$ ( $p=.034)$ and $270^{\circ}(p=.020)$ trials. No other main effects or interactions reached significance.

\section{Errors}

Figure 2B shows mean proportions of errors and standard errors for each condition as a function of rotation magnitude. The participants made significantly fewer errors in the tactile condition $(M=12 \%)$ than in the control condition $(M=20 \%)[F(1,21)=6.04, p<.023]$. We also found a significant effect of rotation $[F(3,63)=19.03, p<.0001]$. Post hoc linear comparisons revealed an error function similar to that for RTs. Errors increased from $0^{\circ}$ to $90^{\circ}(p<$ .003 ) and from $180^{\circ}$ to $270^{\circ}(p<.007)$; however, we found no difference in errors between $90^{\circ}$ and $180^{\circ}(p=.539)$. No other main effects or interactions reached significance.

\section{Discussion}

As was predicted, the participants were faster in the tactile condition than in the control condition. These results are similar to those reported by Wraga et al. (2000) using a between-subjects comparison of control and tactile conditions. Thus, the present within-subjects paradigm we employed was successful at facilitating the participants' mental rotation of a handheld object through passive tactile feedback. In fact, the results exceeded our expectations for improved performance. Not only did passive tactile feedback for the rotating object improve RTs, as compared with the control condition, it also improved accuracy. These findings support the hypothesis that tactile information influences mental rotation performance.

As is evidenced in the main effects of rotation magnitude found, both RT and error functions reflected general monotonic increases typical of imagined object rotation tasks (Shepard \& Cooper, 1982; Shepard \& Metzler, 1971). 

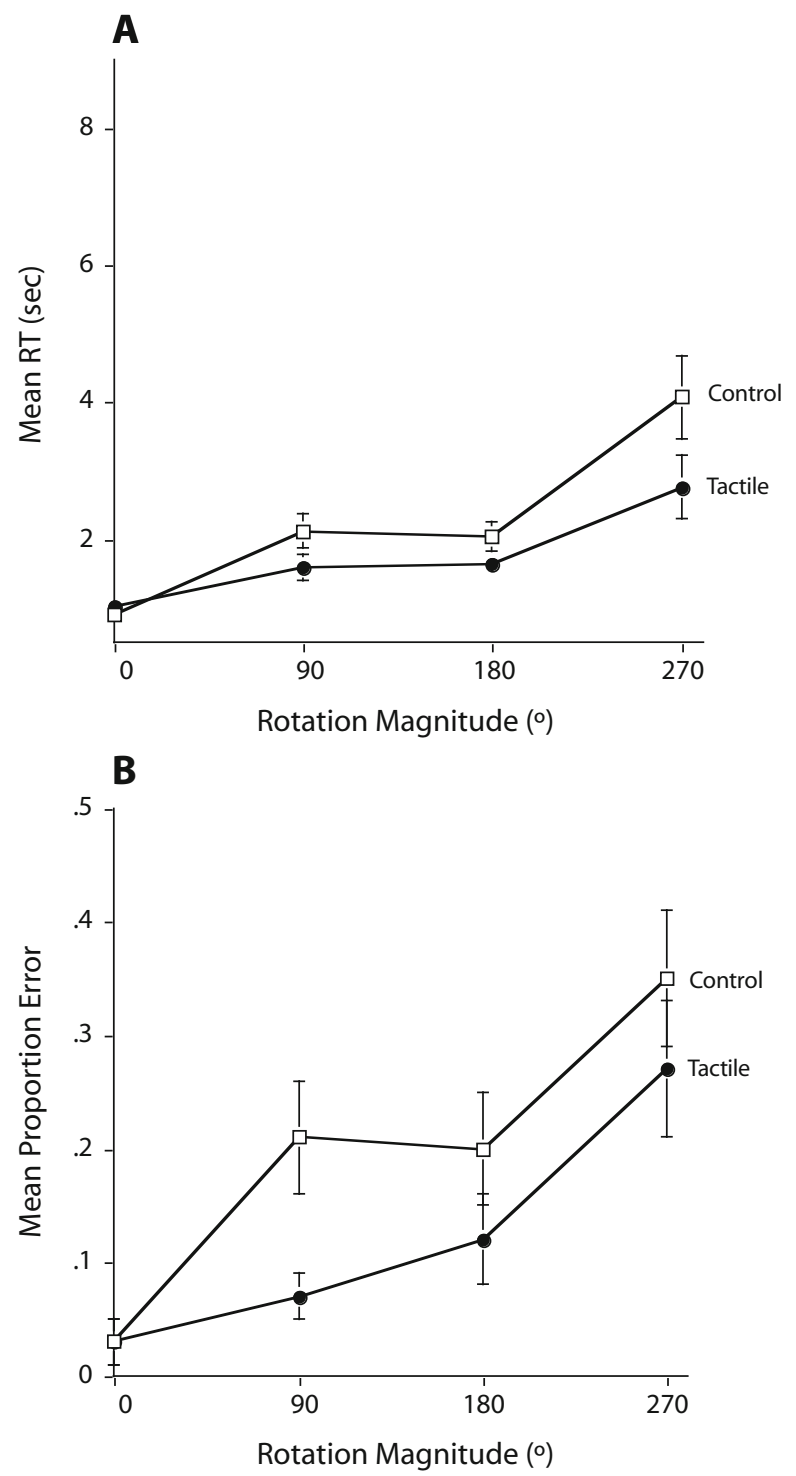

Figure 2. Mean response times (RTs) and standard errors (A) and mean proportions of errors and standard errors (B) for the control and tactile conditions, as a function of rotation magnitude, in Experiment 1.

However, post hoc linear comparisons revealed a flattening of the RT and error functions between $90^{\circ}$ and $180^{\circ}$, which Wraga et al. (2000) also found for performance of a similar task. Wraga et al. (2000) attributed the flatline RT values between $90^{\circ}$ and $180^{\circ}$ rotations to the fact that participants may employ a nonrotational symmetry reversal strategy for $180^{\circ}$ rotations. This interpretation applies to both conditions in the present experiment. The shape of the RT function, combined with the results of the condition $\times$ rotation magnitude interaction, which revealed a minimal impact of the tactile manipulation on $0^{\circ}$ and $180^{\circ}$ trials, sheds light on the nature of the influence of passive tactile feedback on mental rotation performance. The absence of an effect of tactile information for $180^{\circ}$ RT trials suggests that the employment of nonrotational strategies occurs fairly early in the decision process, so that the tactile feedback for the rotating object is effectively ignored. A similar effect was found for $0^{\circ}$ trials, which required no rotation and included static tactile information that was of little benefit to updating the object. In contrast, the tactile manipulation had its greatest effect on $90^{\circ}$ and $270^{\circ}$ rotation trials, which were not easily prone to nonrotational strategies. Theses findings suggest that mental rotation is facilitated by continuous tactile information. However, alternative hypotheses regarding discrete tactile feedback and the precise nature of the tactile facilitation cannot be ruled out at this time. Experiment 2 was designed to address the former issue, and Experiments 3 and 4 were designed to address the latter issue.

\section{EXPERIMENT 2}

Having established the validity of the within-subjects paradigm for manipulating tactile information, we next explored the specific factors of the tactile transformation that contributed to the participants' facilitated mental rotation performance. Wraga et al. (2000) previously had proposed that the presence of tactile information during mental rotation allowed for a more cohesive transformation of the object's internal representation through a continuous, online transformation of the object. However, it also is possible that the specific start- and endpoints of the rotation event provided sufficient information to inform the internal representation of the object. Experiment 2 was designed to examine this issue further. Using a new group of participants, we compared performance in Experiment 1's control condition (stationary object) with performance in a discrete tactile condition in which the experimenter merely moved the object from the startpoint of the rotation event to the endpoint of the rotation event. We predicted that the effect of the discrete tactile manipulation would be negligible, as compared with that in the control condition, and would be reduced, as compared with effects in the tactile condition in Experiment 1.

\section{Method}

\section{Participants}

Twenty-three Smith College undergraduate females participated in the experiment as part of a research credit requirement. The data of 1 additional participant were excluded from the experiment for having more than a $50 \%$ error rate in at least one condition. All the participants were tested individually and were unaware of the hypothesis being tested.

\section{Materials}

See the General Method section.

\section{Procedure}

The procedure was the same as that in Experiment 1, except that the two conditions we compared within subjects, using a new set of participants, were control (see the General Method section) and discrete tactile. In the latter, the participants received only tactile information for the start- and endpoints of the object's prescribed rotation on their palm. At the onset of each trial, the object lay stationary on the participant's upturned palm in the orientation in which the participant had learned its components. For each trial, the experimenter administered the discrete tactile information by picking up the object and placing it back on the participant's palm in the ending 
rotation position, in time with the verbal utterance of the prescribed rotation magnitude. At the end of the trial, the experimenter picked the object up and rotated it in the air to its starting orientation before placing it back on the participant's palm for the next trial.

\section{Design}

See the General Method section.

\section{Results}

\section{Response Times}

Figure 3A shows mean RTs and standard errors for each condition as a function of rotation magnitude. The participants were marginally slower in the discrete tactile condition $(M=2.84 \mathrm{sec})$ than in the control condition $(M=2.48 \mathrm{sec})[F(1,21)=3.23, p=.087]$. The ANOVA yielded a significant effect of rotation $[F(3,63)=32.15$, $p<.0001]$. Post hoc linear comparisons revealed that RTs increased between all degrees $\left(0^{\circ}\right.$ to $90^{\circ}, p<.0001 ; 90^{\circ}$ to $180^{\circ}, p<.008 ; 180^{\circ}$ to $\left.270^{\circ}, p<.0001\right)$.

Between-experiments analysis. For a more direct test of our prediction, we compared the magnitude of Experiment 2's discrete tactile effect with that of the continuous tactile effect in Experiment 1. To achieve this, we calculated the signed difference in RTs between conditions (control - tactile) for each participant in each experiment at each rotation magnitude, expressed as a proportion of the corresponding control RT score (i.e., control $_{90^{\circ}}-$ tactile $_{90^{\circ}} /$ control $_{90^{\circ}}$. This calculation yielded the signed value of the tactile condition independently of the raw RT scores in each experiment, thus controlling for any between-group variability. These scores were submitted to a 2 (experiment) $\times 3$ (rotation magnitude) ANOVA. We found a significantly greater effect in the tactile condition in Experiment $1(M=+0.02)$, as compared with that in Experiment $2(M=-0.43)[F(1,44)=11.95, p<.001]$. No other main effects or interactions were significant.

\section{Errors}

Figure 3B shows mean proportions of errors and standard errors for each condition as a function of rotation magnitude. The participants were similarly accurate in both conditions (control, $M=9 \%$; discrete tactile, $M=$ $11 \% ; p=.550)$. The ANOVA yielded a significant effect of task order $[F(1,21)=5.65, p<.027]$. Post hoc comparisons revealed that the participants who performed the discrete condition last tended to have higher overall error rates than did those who performed it first. We also found a significant effect of rotation magnitude $[F(3,63)=$ $33.11, p<.0001]$. Post hoc linear comparisons revealed that errors marginally increased from $0^{\circ}$ to $90^{\circ}(p=.057)$ and increased from $180^{\circ}$ to $270^{\circ}(p<.0001)$ but did not differ between $90^{\circ}$ and $180^{\circ}(p=.589)$. No other main effects or interactions reached significance.

Between-experiments analysis. To compare the magnitude of the discrete tactile effect in Experiment 2 with that in the continuous tactile effect in Experiment 1, we calculated the signed difference in percentages of errors between conditions (control - tactile) for each participant in each experiment at each rotation magnitude. These scores were submitted to a 2 (experiment) $\times 3$ (rotation magni-

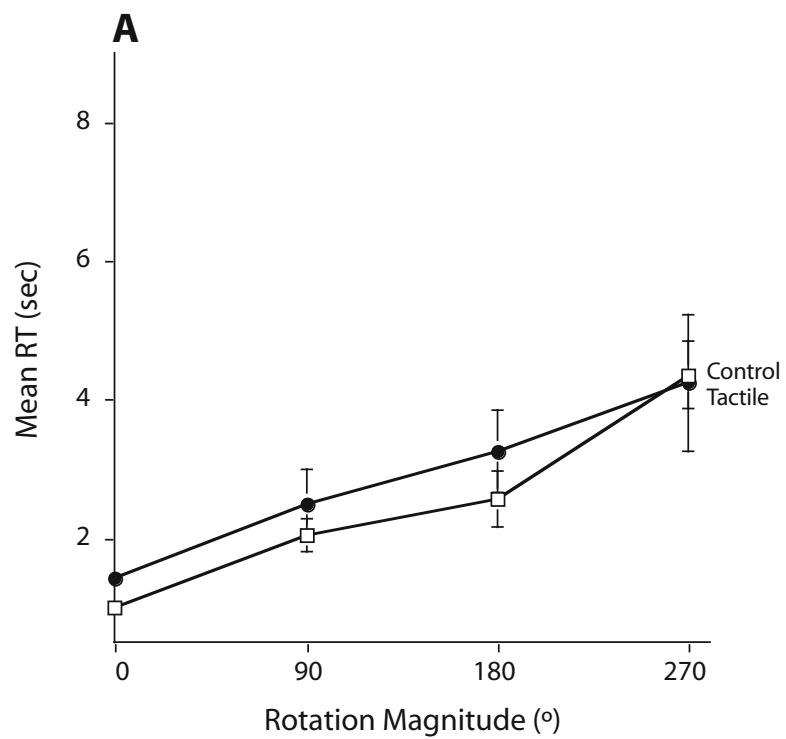

B

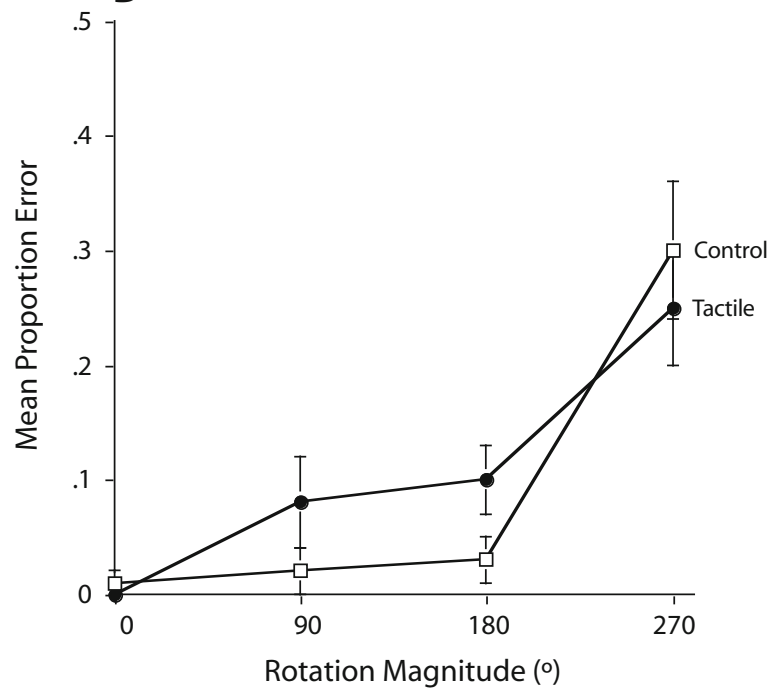

Figure 3. Mean response times (RTs) and standard errors (A) and mean proportions of errors and standard errors (B) for the control and tactile conditions, as a function of rotation magnitude, in Experiment 2.

tude) ANOVA. We found a significantly greater effect of the tactile condition in Experiment $1(M=+0.29)$ than of that in Experiment $2(M=-0.05)[F(1,44)=5.29, p<$ .026]. No other effects or interactions were significant.

\section{Discussion}

As was predicted, the presence of discrete tactile information for the start- and endpoints of the object's rotation path was not sufficient to improve mental rotation performance beyond that in the stationary control condition. In fact, the trend was in the opposite direction, with performance in the discrete tactile condition slightly (albeit not significantly) worse than that in the control condition, for both RTs and errors. Furthermore, the betweenexperiments analyses of RTs and errors confirmed that the 
discrete tactile manipulation in Experiment 2 had a significantly smaller impact on improving mental rotation performance than did the continuous tactile manipulation in Experiment 1. These findings provide clear empirical evidence for eliminating discrete tactile information as a possible factor in facilitating mental rotation performance.

\section{EXPERIMENT 3}

Although the results of Experiment 2 preclude the possibility that discrete tactile information facilitates mental rotation performance, at least one plausible alternative account remains. Continuous tactile feedback, which is a perceptual source of information, may be sufficient for the facilitated performance we found, independently of any influence on the object's internal representation. We explored this issue further in Experiment 3, using a variation of our mental rotation task. For the test objects, we mounted puzzle pieces identical to those in the previous experiments onto circular disks. In this way, the continuous haptic information felt during object rotation was decoupled from the visual information representing the objects' parts (ambiguous tactile condition). We compared performance in the ambiguous tactile condition with that in the stationary object control conditions in Experiments 1 and 2. We predicted that the ambiguous tactile manipulation would not improve performance, relative to that in the control condition.

\section{Method}

\section{Participants}

Twenty-six Smith College undergraduate females participated in the experiment as part of a research credit requirement. The data of 5 additional participants were excluded from the experiment for having more than a $50 \%$ error rate in at least one condition. All the participants were tested individually and were unaware of the hypothesis being tested.

\section{Materials}

The objects used were identical to those in Experiment 1, except that each test object was mounted onto a $9-\mathrm{cm}$-diameter, $1.25-\mathrm{cm}$ thick wooden disk.

\section{Procedure}

The procedure was the same as that in Experiment 1, except that the two conditions we compared within subjects were control (see the General Method section) and ambiguous tactile. In the latter, the object-on-disk was rotated on the participant's upturned palm so that she received tactile information for the disk only, which was independent of the shape of the test object. At the onset of each trial, the object lay stationary on the participant's upturned palm in the orientation in which she had learned its components. For each trial, the experimenter administered the ambiguous tactile information by rotating the knob of the test object so that the entire object-on-disk rotated in time with the verbal utterance of the prescribed rotation magnitude. At the end of the trial, the experimenter picked up the object-on-disk and rotated it in the air to its starting orientation before placing it back on the participant's palm for the next trial.

\section{Design}

See the General Method section.

\section{Results}

\section{Response Times}

Figure 4A shows mean RTs and standard errors for each condition as a function of rotation magnitude. The participants were significantly slower in the ambiguous tactile condition $(M=5.69 \mathrm{sec})$ than in the control condition $(M=4.83 \mathrm{sec})[F(1,24)=11.62, p<.002]$. The ANOVA yielded a significant effect of rotation magnitude $[F(3,72)=45.00, p<.0001]$. Post hoc linear comparisons revealed that RTs increased from $0^{\circ}$ to $90^{\circ}$ ( $p<$ $.0001)$ and from $180^{\circ}$ to $270^{\circ}(p<.0001)$ but did not differ between $90^{\circ}$ and $180^{\circ}(p=.732)$. No other main effects or interactions reached significance.

\section{Errors}

Figure 4B shows mean proportions of errors and standard errors for each condition as a function of rotation magnitude. As in the previous experiment, the participants were similarly accurate in both conditions (control, $M=16 \%$; ambiguous tactile, $M=18 \% ; p=.693$ ).
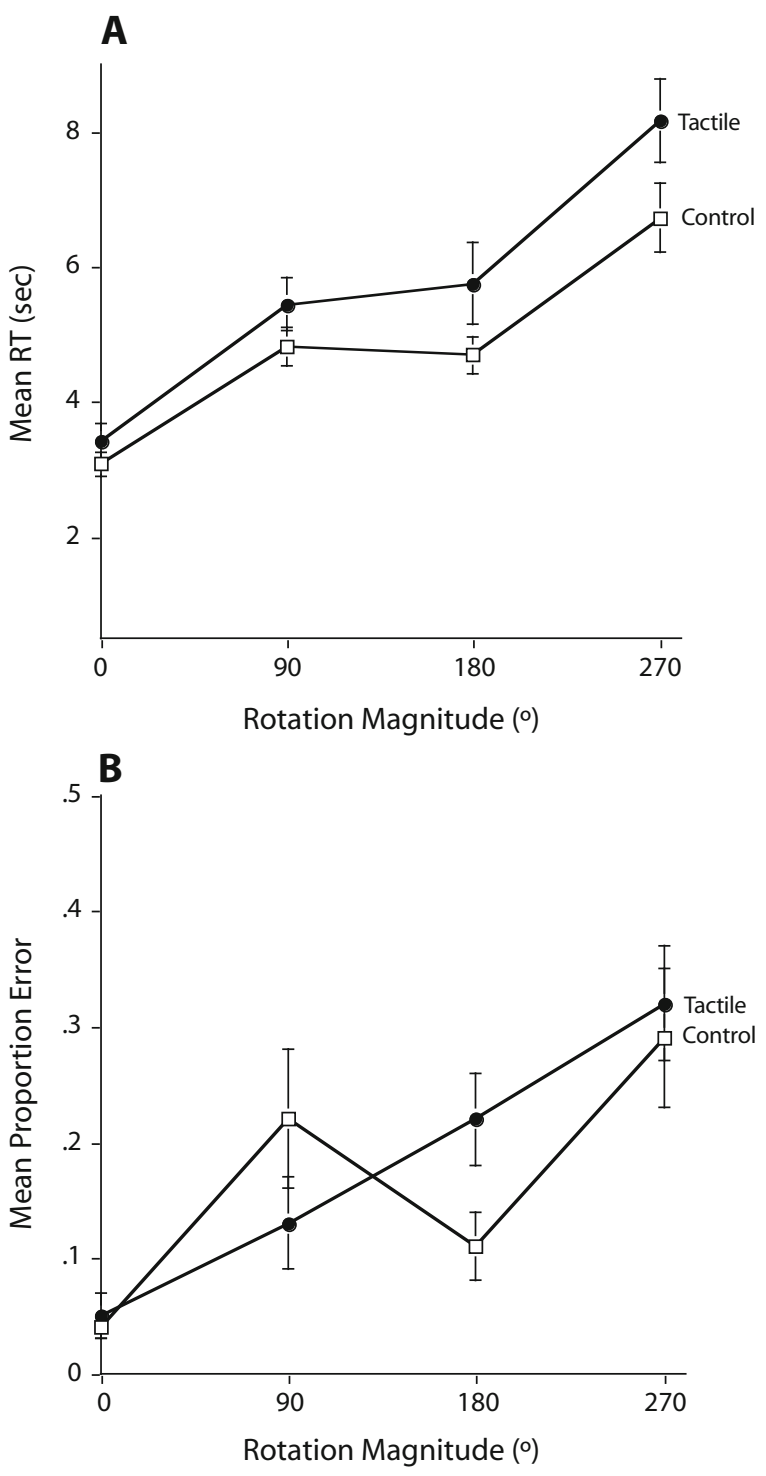

Figure 4. Mean response times (RTs) and standard errors (A) and mean proportions of errors and standard errors (B) for the aligned and misaligned conditions, as a function of rotation magnitude, in Experiment 3. 
We also found a significant effect of rotation magnitude $[F(3,72)=14.00, p<.0001]$. Post hoc linear comparisons revealed that errors increased from $0^{\circ}$ to $90^{\circ}(p=$ $.004)$ and increased from $180^{\circ}$ to $270^{\circ}(p<.0001)$ but did not differ between $90^{\circ}$ and $180^{\circ}(p=.805)$. No other main effects or interactions reached significance.

\section{Discussion}

The presence of continuous tactile information dissociated from object shape did not improve mental rotation performance, relative to that in the stationary control condition, and, in fact, made it worse. Performance in the ambiguous tactile condition was significantly slower than that in the control condition; a similar (albeit nonsignificant) trend occurred for errors. These findings suggest that the facilitated mental rotation performance found previously with our haptic manipulation occurred through enhancement of participants' internal representations of the rotating objects. We explored this issue further in Experiment 4.

\section{EXPERIMENT 4}

Experiment 4 was designed to provide a more direct test of the influence of continuous tactile feedback on mental rotation performance. The participants performed the mental rotation task under two experimental conditions, both of which involved continuous tactile information. The aligned condition was identical to the tactile condition in Experiment 1. The misaligned condition involved trials in which the tactile information for the object was discrepant from the participants' imagined object movement. In the latter condition, the experimenter rotated the object either less or more than the prescribed rotation amount, and the participant's task was to ignore the tactile feedback and to imagine rotating the object the prescribed number of degrees. Previous researchers have used a similar misalignment manipulation in conjunction with imagined rotations of one's body, to demonstrate how physical rotation of the body "automatically" updates object locations with respect to the body without cognitive effort and/or volitional control (e.g., Farrell \& Robertson, 1998; Rieser, 1989). In the present study, however, we viewed the misaligned manipulation as eliciting interference effects within a shared resource (e.g., Brooks, 1968), rather than automatic updating. Given the apparent link between tactile processing and visual imagery, we predicted that the discrepant tactile feedback in the misaligned condition would interfere with the participant's internal representation of the rotating object. This would produce poorer mental rotation performance, relative to that in the aligned condition.

\section{Method}

\section{Participants}

Twenty-three Smith College undergraduate females participated in the experiment as part of a research credit requirement. The data of 1 additional participant were excluded from the experiment for having more than a $50 \%$ error rate in at least one condition. All the participants were tested individually and were unaware of the hypothesis being tested.

\section{Materials}

See the General Method section.

\section{Procedure}

The procedure was the same as that in Experiment 1, except that the two conditions we compared within subjects were aligned (identical to the tactile condition in Experiment 1) and misaligned. For each trial in the misaligned condition, the participant received continuous tactile information in an amount discrepant from that of target rotation. For example, if the target rotation of the object was $90^{\circ}$, the experimenter might rotate the object $270^{\circ}$ on the participant's palm, for a disparity of $+180^{\circ}$. The experimenter always rotated the object in a clockwise direction. The amount of rotation discrepancy ranged from $-270^{\circ}$ to $+270^{\circ}$, in $90^{\circ}$ increments, with the exception of a $0^{\circ}$ disparity (the latter would be equivalent to the prescribed amount of rotation and, thus, would constitute a negation of the misaligned effect). As in the previous experiments, the experimenter always lifted up the object and then placed it back at its starting position on the participant's palm at the end of each trial.

\section{Design}

The design was similar to that in the previous experiments, apart from one addition. In the misaligned condition, the magnitude of the discrepant physical rotation (i.e., $-270,-180,-90,+90,+180$, or +270 ) occurred quasirandomly across trials. Because of the physical constraints imposed by the object configurations and by the clockwise direction of the discrepant rotation, it was not possible to evenly distribute discrepancy values across the four rotation conditions. For $0^{\circ}$ rotations, the magnitudes of discrepancy consisted of $+90^{\circ}$, $+180^{\circ}$, or $+270^{\circ}$. For $90^{\circ}$ rotations, the magnitudes of discrepancy consisted of $\pm 90^{\circ},+180^{\circ}$, or $+270^{\circ}$. For $180^{\circ}$ rotations, the magnitudes of discrepancy consisted of $\pm 90^{\circ}$ or $\pm 180^{\circ}$. For $270^{\circ}$ rotations, the magnitudes of discrepancy consisted of $\pm 90^{\circ},-180^{\circ}$, or $-270^{\circ}$. However, the total magnitude of discrepant rotation within each class of rotation was held equivalent.

\section{Results}

\section{Response Times}

Figure 5A shows mean RTs and standard errors for each tactile condition, as a function of rotation magnitude. The participants were significantly faster in the aligned condition $(M=2.34 \mathrm{sec})$ than in the misaligned condition $(M=3.12 \mathrm{sec})[F(1,21)=8.71, p<.008]$. The ANOVA also yielded a significant effect of rotation magnitude $[F(3,63)=33.62, p<.0001]$. Post hoc linear comparisons revealed an increase in RTs from $0^{\circ}$ to $90^{\circ}(p<$ $.0001)$ and from $180^{\circ}$ to $270^{\circ}(p<.001)$, but not between $90^{\circ}$ and $180^{\circ}(p=.721)$. We also found a significant condition $\times$ rotation magnitude interaction $[F(3,63)=4.50$, $p<.006]$. Contrast comparisons indicated that poorer performance in the misaligned condition occurred most significantly for $90^{\circ}(p=.066)$ and $270^{\circ}(p=.002)$ trials. Moreover, the deficit in performance for $270^{\circ}$ rotations in the misaligned condition was more than twice $(M=$ $1.83 \mathrm{sec})$ that of the deficit for $90^{\circ}$ rotations in the same condition $(M=0.89 \mathrm{sec})$. No other main effects or interactions reached significance.

\section{Errors}

Figure 5B shows mean proportions of errors and standard errors for each tactile condition as a function of rotation magnitude. The participants were similarly accurate in both conditions (aligned, $M=9 \%$; misaligned, $M=$ $10 \% ; p=.765$ ). We found a significant effect of rotation magnitude only $[F(3,63)=22.38, p<.0001]$. Post hoc linear comparisons revealed that errors increased from $0^{\circ}$ to $90^{\circ}(p=.001)$ and from $180^{\circ}$ to $270^{\circ}(p<.0001)$ but 
A

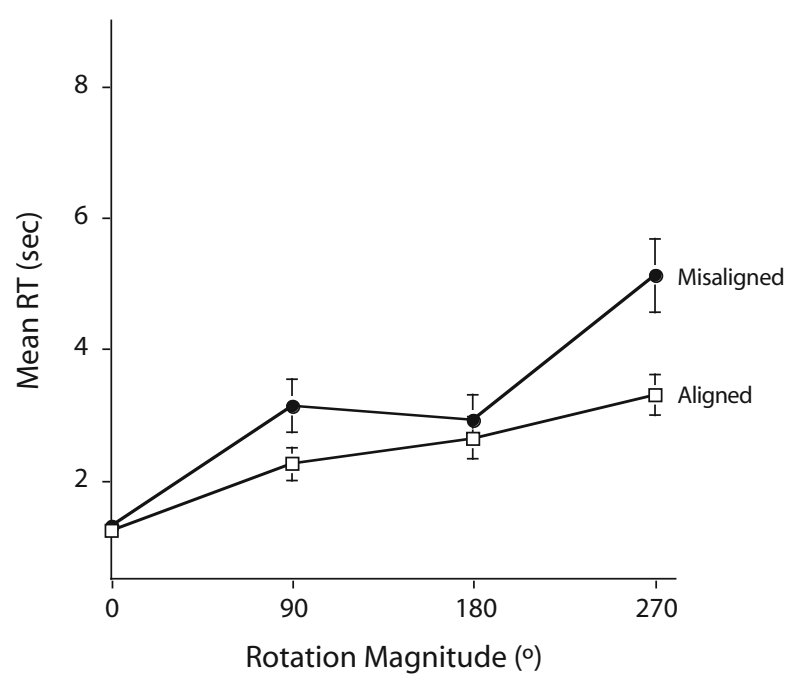

B

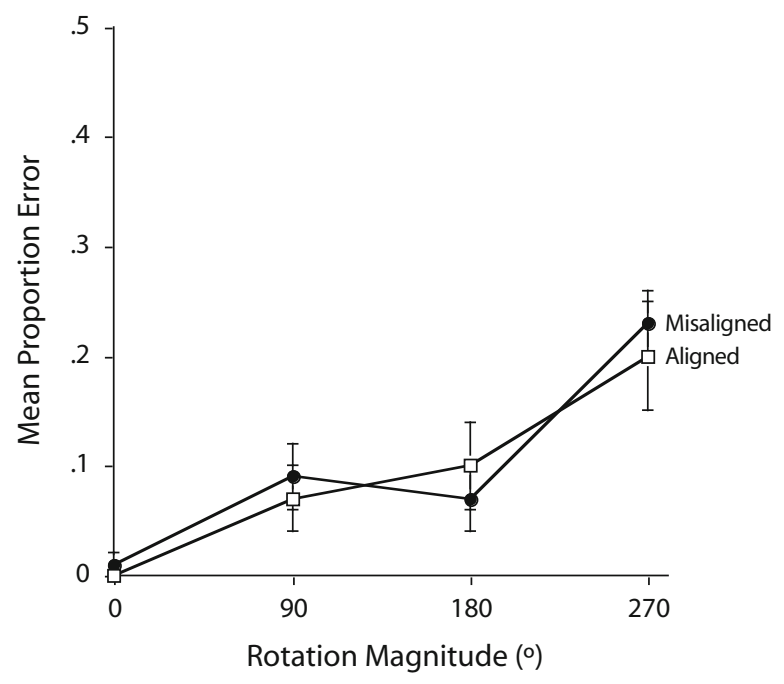

Figure 5. Mean response times (RTs) and standard errors (A) and mean proportions of errors and standard errors (B) for the aligned and misaligned conditions, as a function of rotation magnitude, in Experiment 4.

did not differ between $90^{\circ}$ and $180^{\circ}(p=.897)$. No other main effects or interactions reached significance.

\section{Discussion}

As was predicted, performance was worse in the misaligned condition than in the aligned condition, although this deficit was revealed in RTs only, and not in errors. As in the previous experiments, the data for both response measures showed a general monotonic increase as a function of rotation magnitude. However, the effects of the misaligned manipulation on RTs (as assessed in comparison with aligned RTs) were not distributed evenly. The greatest effect of the misaligned manipulation occurred for $90^{\circ}$ and, particularly, $270^{\circ}$ rotation trials in a pattern similar to the facilitative effect in the tactile condition in Experi- ment 1 . Given that the $0^{\circ}$ rotation trials did not require rotation and that, as was suggested previously, the participants may have used nonrotational strategies for $180^{\circ}$ trials, this finding lends further support to the idea that the holistic nature of continuous tactile information plays a major role in influencing mental rotation performance. This interpretation is further strengthened by the fact that the total magnitude of discrepant rotation administered in the misaligned condition remained constant across each of the four degrees of rotation. The increased impact in the misaligned condition for $270^{\circ}$ trials thus suggests greater interference with the participants' dynamic internal representations, which require more cognitive processing than do $90^{\circ}$ rotations.

A comparison of Figures $2 \mathrm{~A}$ and $5 \mathrm{~A}$ indicates that the participants in the aligned condition in Experiment 4 were somewhat slower and made more errors than did the participants in the identical tactile condition in Experiment 1 . One possibility for this performance difference is that exposure to the misaligned condition in Experiment 4 somehow generalized to all performance; however, the absence of a task order effect in either the RT or the error analysis renders this possibility implausible. A more likely explanation is that the differences are the result of between-group variability across experiments. Such an interpretation underscores the importance of using withinsubjects designs for performance comparisons.

\section{GENERAL DISCUSSION}

In four experiments, we tested the conditions under which passive tactile feedback influences mental rotation of handheld objects. In Experiment 1, we established within subjects that passive tactile information specifying an object's rotation event facilitated the participants' mental rotation performance, as compared with a control condition in which the object remained stationary during mental rotation performance. Experiment 2 demonstrated that tactile information specifying the discrete start- and endpoints of the rotating object was not sufficient for improving mental rotation performance beyond that in the stationary control condition. Experiment 3 demonstrated that continuous tactile information independent of object shape also was not sufficient for improving mental rotation performance and actually hindered performance. Experiment 4 revealed that continuous tactile feedback also can hinder mental rotation performance when the feedback is misaligned from that involved in the dynamic internal representation of the object.

The results of these experiments add to the findings of a growing list of studies demonstrating external influences on visual mental rotation performance (e.g., Corballis \& Blackman, 1990; Heil, Bajrić, Rösler, \& Hennighausen, 1997; Jolicœur, Corballis, \& Lawson, 1998). More important, our results also replicate the finding that tactile information for an object's rotation facilitates mental rotation performance (Wraga et al., 2000). We previously had proposed that tactile information specifying a rotating object allowed for a more cohesive internal transformation of the object's spatial components through the con- 
tinuous, online transformation of the object. The results of the present study provide more distinctive evidence supporting this hypothesis. In Experiment 1, improved mental rotation performance in the presence of continuous tactile information was greatest during the "true" rotation trials of $90^{\circ}$ and $270^{\circ}$, suggesting that the continuous nature of the tactile information was the critical factor in facilitating performance. On the other hand, continuous rotation of the object that was out of sync with the participants' imagined rotation hindered performance in a similar pattern, with the worst effects occurring for $90^{\circ}$ and $270^{\circ}$ trials.

These findings suggest a tight coupling between the continuous tactile information specifying a rotating object and the corresponding dynamic representation of the rotating object. This correspondence between haptic and visual modalities is in line with Sathian and colleagues' proposed account of haptic-visual translation for mental rotation (Prather \& Sathian, 2002; Prather et al., 2004; Sathian, 2005). Research explicating the neural mechanisms underlying this relationship is in an incipient stage. Recent neuroimaging studies suggest that visual, as well as spatial, processing areas of the brain may play a role. For example, simple tactile tasks, such as the discrimination of the orientation of grating patterns by touch, have been found to activate visual-processing regions of the human brain such as the parieto-occipital junction (POJ; Sathian, Zangaladze, Hoffman, \& Grafton, 1997). Moreover, such activation plays a functional role in tactile pattern discrimination. When transcranial magnetic stimulation was applied to participants' POJ while they performed the tactile pattern discrimination task, performance was impaired (Sathian \& Zangaladze, 2002). More recently, Prather et al. (2004) used positron emission tomography to ascertain the brain regions associated with mental rotation of unseen letter shapes pressed onto participants' fingers. When compared with a verbal control, this task was found to activate the anterior intraparietal sulcus, which is part of the dorsal visual pathway. Prather et al. proposed that this region may be the site where tactile information is translated into visual information. If this assertion is correct, we would expect the anterior intraparietal sulcus to play a key role in performance in the tactile condition of the present study. This is an issue for future research.

In summary, our findings indicate that passive tactile feedback facilitates mental rotation performance. Moreover, it is the continuous aspect of the tactile rotation, and not its discrete start- and endpoints, that contributes to performance facilitation. These findings suggest a tight coupling between tactile information specifying a rotating object and the corresponding dynamic internal representation of the rotating object.

\section{AUTHOR NOTE}

This research was supported by NSF Grant BCS-0517939 to the first author. We thank three anonymous reviewers for their comments on an earlier draft of the manuscript. We also thank Emma Jane and Francis Konkoly, whose puzzle was the inspiration for the stimuli used in this study. Correspondence concerning this article should be addressed to M. Wraga, Department of Psychology, Smith College, Northampton, MA 01063 (e-mail: mwraga@ smith.edu).

\section{REFERENCES}

BrooKs, L. R. (1968). Spatial and verbal components of the act of recall. Canadian Journal of Psychology, 22, 349-368.

Carpenter, P. A., \& Eisenberg, P. (1978). Mental rotation and the frame of reference in blind and sighted individuals. Perception \& Psychophysics, 23, 117-124.

Cohen, M. S., Kosslyn, S. M., Breiter, H. C., Digirolamo, G. J., Thompson, W. L., Anderson, A. K., ET AL. (1996). Changes in cortical activity during mental rotation: A mapping study using functional MRI. Brain, 119, 89-100.

Corballis, M. C., \& Blackman, A. R. (1990). The effect of apparent motion on mental rotation. Memory \& Cognition, 18, 551-555.

Corballis, M. C., \& McMaster, H. (1996). The roles of stimulusresponse compatibility and mental rotation in mirror-image and leftright decisions. Canadian Journal of Experimental Psychology, 50, 397-401.

Creem, S. H., Wraga, M., \& Proffitt, D. R. (2001). Imagining physically impossible self-rotations: Geometry is more important than gravity. Cognition, 81, 41-64.

Dellantonio, A., \& Spagnolo, F. (1990). Mental rotation of tactual stimuli. Acta Psychologica, 73, 245-257.

Farrell, M. J., \& Robertson, I. H. (1998). Mental rotation and the automatic updating of body-centered spatial relationships. Journal of Experimental Psychology: Learning, Memory, \& Cognition, 24, 227-233.

Heil, M., Bajrić, J., Rösler, F., \& Hennighausen, E. (1997). A rotation aftereffect changes both the speed and the preferred direction of mental rotation. Journal of Experimental Psychology: Human Perception \& Performance, 23, 681-692.

Jolicceur, P., \& CAVANaGh, P. (1992). Mental rotation, physical rotation, and surface media. Journal of Experimental Psychology: Human Perception \& Performance, 18, 371-384.

Jolicceur, P., Corballis, M. C., \& Lawson, R. (1998). The influence of perceived rotary motion on the recognition of rotated objects. Psychonomic Bulletin \& Review, 5, 140-146.

Kosslyn, S. M., DiGirolamo, G. J., Thompson, W. L., \& Alpert, N. M. (1998). Mental rotation of objects versus hands: Neural mechanisms revealed by positron emission tomography. Psychophysiology, 35, 151-161.

Lederman, S. J., \& KLATZKY, R. L. (1997). Relative availability of surface and object properties during early haptic processing. Journal of Experimental Psychology: Human Perception \& Performance, 23, 1690-1707.

Loomis, J. M., \& Lederman, S. J. (1986). Tactual perception. In K. R. Boff, L. Kaufman, \& J. P. Thomas (Eds.), Handbook of perception and human performance: Vol. II. Cognitive processes and performance (pp. 31.1-31.41). New York: Wiley.

MARMOR, G. S., \& ZABACK, L. A. (1976). Mental rotation by the blind: Does mental rotation depend on visual imagery? Journal of Experimental Psychology: Human Perception \& Performance, 2, 515-521.

PARSONS, L. M. (1987). Imagined spatial transformations of one's hands and feet. Cognitive Psychology, 19, 178-241.

Prather, S. C., \& Sathian, K. (2002). Mental rotation of tactile stimuli. Cognitive Brain Research, 14, 91-98.

Prather, S. C., Votaw, J. R., \& Sathian, K. (2004). Task-specific recruitment of dorsal and ventral visual areas during tactile perception. Neuropsychologia, 42, 1079-1087.

Presson, C. C. (1982). Strategies in spatial reasoning. Journal of Experimental Psychology: Learning, Memory, \& Cognition, 8, 243-251.

PyLYshyn, Z. W. (1973). What the mind's eye tells the mind's brain: A critique of mental imagery. Psychological Bulletin, 80, 1-24.

RIESER, J. J. (1989). Access to knowledge of spatial structure at novel points of observation. Journal of Experimental Psychology: Learning, Memory, \& Cognition, 15, 1157-1165.

Robert, M., \& Chevrier, E. (2003). Does men's advantage in mental rotation persist when real three-dimensional objects are either felt or seen? Memory \& Cognition, 31, 1136-1145.

SATHIAN, K. (2005). Visual cortical activity during tactile perception in the sighted and the visually deprived. Developmental Psychobiology, 46, 279-286.

Sathian, K., \& Zangaladze, A. (2002). Feeling with the mind's eye: 
Contribution of visual cortex to tactile perception. Behavioural Brain Research, 135, 127-132.

Sathian, K., Zangaladze, A., Hoffman, J. M., \& Grafton, S. T. (1997). Feeling with the mind's eye. NeuroReport, 8, 3877-3881.

Shepard, R. N., \& CoOPER, L. A. (1982). Mental images and their transformations. Cambridge, MA: MIT Press.

ShePard, R. N., \& Metzler, J. (1971). Mental rotation of threedimensional objects. Science, 171, 701-703.

Voisin, J., Benoit, G., \& Chapman, C. E. (2002). Haptic discrimination of object shape in humans: Two-dimensional angle discrimination. Experimental Brain Research, 145, 239-250.

Voisin, J., Lamarre, Y., \& Chapman, C. E. (2002). Haptic discrimination of object shape in humans: Contribution of cutaneous and proprioceptive inputs. Experimental Brain Research, 145, 251-260.

Wraga, M., Creem, S. H., \& Proffitt, D. R. (2000). Updating displays after imagined object and viewer rotations. Journal of Experimental Psychology: Learning, Memory, \& Cognition, 26, 151-168.
Wraga, M., Shephard, J. M., Church, J. A., Inati, S., \& Kosslyn, S. M. (2005). Imagined rotations of self versus objects: An fMRI study. Neuropsychologia, 43, 1351-1361.

\section{NOTES}

1. Preliminary analyses of all the experiments indicated that orientation of object with respect to the hand had no impact on the participants' RTs or accuracy. Therefore, we excluded it from the final analyses reported in this article.

2. On the basis of many past studies on mental rotation of objects, we considered RTs to be the primary dependent measure for our task (e.g., Creem, Wraga, \& Proffitt, 2001; Presson, 1982; Shepard \& Cooper, 1982; Shepard \& Metzler, 1971; Wraga et al., 2000).

(Manuscript received August 2, 2006; revision accepted for publication August 15, 2007.) 